\title{
COEFFICIENT ESTIMATES FOR SPECIAL SUBCLASSES OF $k$-FOLD SYMMETRIC BI-UNIVALENT FUNCTIONS
}

\author{
WAGGAS GALIB ATSHAN, SIBEL YALÇIN AND RASHA ABBAS HADI
}

\begin{abstract}
In the present paper, we consider two new subclasses $\mathcal{N}_{\Sigma_{k}}(\mu, \alpha, \tau)$ and $\mathcal{N}_{\Sigma_{k}}(\mu, \beta, \tau)$ of $\Sigma_{k}$ consisting of analytic and $k$-fold symmetric bi-univalent functions defined in the open unit disc $\mathcal{U}=\{z: z \in \mathbb{C}$ and $|z|<1\}$. For functions belonging to the two classes introduced here, we derive their normalized forms. Furthermore, we find estimates of the initial coefficients $\left|a_{k+1}\right|$ and $\left|a_{2 k+1}\right|$ for these functions. Several related classes are also considered and connections to previously known results are made.
\end{abstract}

\section{INTRODUCTION}

Let $\mathcal{S}$ denote the family of functions analytic in the open unit disc

$$
\mathcal{U}=\{z: z \in \mathbb{C} \text { and }|z|<1\},
$$

and normalized by the conditions $f(0)=f^{\prime}(0)-1=0$ and having the form:

$$
f(z)=z+\sum_{j=2}^{\infty} a_{j} z^{j} .
$$

Also, let $\mathcal{A}$ denote the subclass of functions in $\mathcal{S}$ which are univalent in $\mathcal{U}$. The Koebe One Quarter Theorem (e.g., see [3]) ensures that the image of $\mathcal{U}$ under every function $f(z) \in \mathcal{S}$ contains the disk of radius $\frac{1}{4}$. It is well known that every function $f$ has an inverse $f^{-1}$ satisfying:

$$
f^{-1}(f(z))=z,(z \in \mathcal{U}) \text { and } f\left(f^{-1}(w)\right)=w,\left(|w|<r_{0}(f) ; r_{0}(f) \geq \frac{1}{4}\right),
$$

where

$$
f^{-1}(w)=g(w)=w-a_{2} w^{2}+\left(2 a_{2}^{2}-a_{3}\right) w^{3}-\left(5 a_{2}^{3}-5 a_{2} a_{3}+a_{4}\right) w^{4}+\cdots .
$$

A function $f \in \mathcal{S}$ is said to be bi-univalent in $\mathcal{U}$ if both $f$ and $f^{-1}$ are univalent in $\mathcal{U}$. Let $\Sigma$ denote the class of all bi-univalent functions in $\mathcal{U}$. Let $\Sigma$ denote the class of all bi-univalent functions in $\mathcal{U}$. Examples of functions in class $\Sigma$ are

$$
h_{1}(z)=\frac{z}{1-z}, h_{2}(z)=-\log (1-z), h_{3}(z)=\frac{1}{2} \log \left(\frac{1+z}{1-z}\right), z \in \mathcal{U} .
$$

$M S C$ (2010): primary 30C45.

Keywords: analytic functions, univalent functions, bi-univalent functions, $k$-fold symmetric, coefficient bounds. 
For each function $f \in \mathcal{A}$, the function $h(z)=\sqrt[k]{f\left(z^{k}\right)},(z \in \mathcal{U}, k \in \mathbb{N})$ is univalent and maps the unit $\operatorname{disc} \mathcal{U}$ into a region with $k$-fold symmetry. A function is said to be $k$-fold symmetric (see $[7,8]$ ) if it has the following normalized form:

$$
f(z)=z+\sum_{j=1}^{\infty} a_{k j+1} z^{k j+1}, \quad(z \in \mathcal{U}, k \in \mathbb{N}) .
$$

We denote $\mathcal{S}_{k}$ the class of $k$-fold symmetric univalent functions in $\mathcal{U}$, which are normalized by the series expansion (1.3). In fact, the functions in the class $\mathcal{A}$ are one-fold symmetric.

Analogously to the concept of $k$-fold symmetric univalent functions, their study gives some important results, such as the one saying that a function $f \in \Sigma$ generates a $k$-fold symmetric bi-univalent function for each $k \in \mathbb{N}$. Furthermore, for the normalized form of $f$ given by (1.3), we obtain the series expansion for $f^{-1}$ as follows:

$$
\begin{aligned}
g(w)=w & -a_{k+1} w^{k+1}+\left[(k+1) a_{k+1}^{2}-a_{2 k+1}\right] w^{2 k+1} \\
& -\left[\frac{1}{2}(k+1)(3 k+2) a_{k+1}^{3}-(3 k+2) a_{k+1} a_{2 k+1}+a_{3 k+1}\right] w^{3 k+1}+\cdots,
\end{aligned}
$$

where $f^{-1}=g$. We denote by $\Sigma_{k}$ the class of $k$-fold symmetric bi-univalent functions in $\mathcal{U}$. For $k=1$, the formula (1.4) coincides with the formula (1.2) of the class $\Sigma$.

Some examples of $k$-fold symmetric bi-univalent functions are given as follows:

$$
\left(\frac{z^{k}}{1-z^{k}}\right)^{\frac{1}{k}},\left[-\log \left(1-z^{k}\right)\right]^{\frac{1}{k}},\left[\frac{1}{2} \log \left(\frac{1+z^{k}}{1-z^{k}}\right)\right]^{\frac{1}{k}} .
$$

Recently, many authors investigated bounds for the various subclasses of $k$-fold symmetric bi-univalent functions (see $[1,2,4,10,11,13])$. This work aims to introduce the new subclasses $\mathcal{N}_{\Sigma_{k}}(\mu, \alpha, \tau)$ and $\mathcal{N}_{\Sigma_{k}}(\mu, \beta, \tau)$ of $\Sigma_{k}$ and find estimates of the coefficients $\left|a_{k+1}\right|$ and $\left|a_{2 k+1}\right|$ for functions in each of these new subclasses.

\section{Main Results}

Definition 2.1. A function $f \in \Sigma_{k}$ given by (1.3) is said to be in the class $\mathcal{N}_{\Sigma_{k}}(\mu, \alpha, \tau)$ if the following conditions are satisfied:

$$
\left|\arg \left(1+\frac{1}{\tau}\left[\frac{(1-\mu)\left(z f^{\prime}(z)-f(z)\right)}{(1-\mu) f(z)+\mu z f^{\prime}(z)}\right]\right)\right|<\frac{\alpha \pi}{2}
$$

and

$$
\begin{gathered}
\left|\arg \left(1+\frac{1}{\tau}\left[\frac{(1-\mu)\left(w g^{\prime}(w)-g(w)\right)}{(1-\mu) g(w)+\mu w g^{\prime}(w)}\right]\right)\right|<\frac{\alpha \pi}{2} \\
(0<\alpha \leq 1 ; 0 \leq \mu<1 ; \tau \in \mathbb{C} \backslash\{0\} ; z, w \in \mathcal{U}),
\end{gathered}
$$

where the function $g=f^{-1}$ is given by (1.4). 
Definition 2.2. A function $f \in \Sigma_{k}$ given by (1.3) is said to be in the class $\mathcal{N}_{\Sigma_{k}}(\mu, \beta, \tau)$ if the following conditions are satisfied:

$$
\operatorname{Re}\left(1+\frac{1}{\tau}\left[\frac{(1-\mu)\left(z f^{\prime}(z)-f(z)\right)}{(1-\mu) f(z)+\mu z f^{\prime}(z)}\right]\right)>\beta
$$

and

$$
\begin{aligned}
& \operatorname{Re}\left(1+\frac{1}{\tau}\left[\frac{(1-\mu)\left(w g^{\prime}(w)-g(w)\right)}{(1-\mu) g(w)+\mu w g^{\prime}(w)}\right]\right)>\beta \\
& (0 \leq \beta<1 ; 0 \leq \mu<1 ; \tau \in \mathbb{C} \backslash\{0\} ; z, w \in \mathcal{U}),
\end{aligned}
$$

where the function $g=f^{-1}$ is given by (1.4).

Lemma 2.3. (See [6]) If $h \in \mathcal{P}$, then $\left|c_{k}\right| \leq 2$ for each $k \in \mathbb{N}$, where $\mathcal{P}$ is the family of all functions $h$ analytic in $\mathcal{U}$, for which

$$
\operatorname{Re}(h(z))>0,(z \in \mathcal{U})
$$

where

$$
h(z)=1+c_{1} z+c_{2} z^{2}+\cdots,(z \in \mathcal{U}) .
$$

Theorem 2.4. Let $f \in \mathcal{N}_{\Sigma_{k}}(\mu, \alpha, \tau)(0<\alpha \leq 1 ; 0 \leq \mu<1 ; \tau \in \mathbb{C} \backslash\{0\})$ be of the form (1.3). Then

$$
\left|a_{k+1}\right| \leq \frac{2 \alpha|\tau|}{k(1-\mu) \sqrt{|1+\alpha(2 \tau-1)|}}
$$

and

$$
\left|a_{2 k+1}\right| \leq \frac{2 \alpha^{2}|\tau|^{2}(k+1)}{k^{2}(1-\mu)^{2}}+\frac{\alpha|\tau|}{k(1-\mu)} .
$$

Proof. It follows from (2.1) and (2.2) that

$$
1+\frac{1}{\tau}\left[\frac{(1-\mu)\left(z f^{\prime}(z)-f(z)\right)}{(1-\mu) f(z)+\mu z f^{\prime}(z)}\right]=[p(z)]^{\alpha}
$$

and

$$
1+\frac{1}{\tau}\left[\frac{(1-\mu)\left(w g^{\prime}(w)-g(w)\right)}{(1-\mu) g(w)+\mu w g^{\prime}(w)}\right]=[q(w)]^{\alpha},
$$

where the functions $p(z)$ and $q(w)$ are in $\mathcal{P}$ and have the following series representations:

and

$$
p(z)=1+p_{k} z^{k}+p_{2 k} z^{2 k}+p_{3 k} z^{3 k}+\ldots
$$

$$
q(w)=1+q_{k} w^{k}+q_{2 k} w^{2 k}+q_{3 k} w^{3 k}+\ldots
$$

Now, equating the coefficients in (2.6) and (2.7), we obtain

and

$$
\begin{gathered}
\frac{k(1-\mu)}{\tau} a_{k+1}=\alpha p_{k}, \\
\frac{(1-\mu) k}{\tau}\left[2 a_{2 k+1}-(1+k \mu) a_{k+1}^{2}\right]=\alpha p_{2 k}+\frac{\alpha(\alpha-1)}{2} p_{k}^{2},
\end{gathered}
$$

$$
\begin{gathered}
-\frac{k(1-\mu)}{\tau} a_{k+1}=\alpha q_{k}, \\
\frac{(1-\mu) k}{\tau}\left[2(k+1) a_{k+1}^{2}-2 a_{2 k+1}-(1+k \mu) a_{k+1}^{2}\right]=\alpha q_{2 k}+\frac{\alpha(\alpha-1)}{2} q_{k}^{2} .
\end{gathered}
$$


From (2.10) and (2.12), we find

$$
p_{k}=-q_{k}
$$

and

$$
2 \frac{k^{2}(1-\mu)^{2} a_{k+1}^{2}}{\tau^{2}}=\alpha^{2}\left(p_{k}^{2}+q_{k}^{2}\right) .
$$

From (2.11), (2.13) and (2.14), we get

$$
\begin{aligned}
\frac{2 k^{2}(1-\mu)^{2} a_{k+1}^{2}}{\tau} & =\alpha\left(p_{2 k}+q_{2 k}\right)+\frac{\alpha(\alpha-1)}{2}\left(p_{k}^{2}+q_{k}^{2}\right) \\
& =\alpha\left(p_{2 k}+q_{2 k}\right)+\frac{(\alpha-1) k^{2}(1-\mu)^{2}}{\alpha \tau^{2}} a_{k+1}^{2}
\end{aligned}
$$

Therefore, we have

$$
a_{k+1}^{2}=\frac{\alpha^{2} \tau^{2}\left(p_{2 k}+q_{2 k}\right)}{k^{2}(1-\mu)^{2}[1+\alpha(2 \tau-1)]} .
$$

Applying Lemma 2.3, for the coefficients $p_{2 k}$ and $q_{2 k}$, we have

$$
\left|a_{k+1}\right| \leq \frac{2 \alpha|\tau|}{k(1-\mu) \sqrt{|1+\alpha(2 \tau-1)|}} .
$$

This gives the desired bound for $\left|a_{k+1}\right|$ as asserted in (2.5). In order to find the bound on $\left|a_{2 k+1}\right|$, by subtracting (2.13) from (2.11), we get

$$
\frac{2 k(1-\mu)}{\tau}\left[2 a_{2 k+1}-(k+1) a_{k+1}^{2}\right]=\alpha\left(p_{2 k}-q_{2 k}\right)+\frac{\alpha(\alpha-1)}{2}\left(p_{k}^{2}-q_{k}^{2}\right) .
$$

It follows from (2.14) and (2.15) that

$$
a_{2 k+1}=\frac{\alpha^{2} \tau^{2}\left(p_{k}^{2}+q_{k}^{2}\right)(k+1)}{4 k^{2}(1-\mu)^{2}}+\frac{\alpha \tau\left(p_{2 k}-q_{2 k}\right)}{4 k(1-\mu)} .
$$

Applying Lemma 2.3 once again for the coefficients $p_{k}, p_{2 k}, q_{k}$ and $q_{2 k}$, we readily obtain

$$
\left|a_{2 k+1}\right| \leq \frac{2 \alpha^{2}|\tau|^{2}(k+1)}{k^{2}(1-\mu)^{2}}+\frac{\alpha|\tau|}{k(1-\mu)} .
$$

The following theorem finds the estimates of the coefficients $\left|a_{k+1}\right|$ and $\left|a_{2 k+1}\right|$ for functions in the class $\mathcal{N}_{\Sigma_{k}}(\mu, \beta, \tau)$.

Theorem 2.5. Let $f \in \mathcal{N}_{\Sigma_{k}}(\mu, \beta, \tau)(0<\beta \leq 1 ; 0 \leq \mu<1 ; \tau \in \mathbb{C} \backslash\{0\})$ be of the form (1.3). Then

$$
\begin{gathered}
\left|a_{k+1}\right| \leq \frac{\sqrt{2|\tau|(1-\beta)}}{k(1-\mu)} \\
\left|a_{2 k+1}\right| \leq \frac{|\tau|^{2}(1-\beta)^{2}(k+1)}{2 k^{2}(1-\mu)^{2}}+\frac{|\tau|(1-\beta)}{k(1-\mu)} .
\end{gathered}
$$

Proof. It follows from (2.3) and (2.4) that

$$
1+\frac{1}{\tau}\left[\frac{(1-\mu)\left(z f^{\prime}(z)-f(z)\right)}{(1-\mu) f(z)+\mu z f^{\prime}(z)}\right]=\beta+(1-\beta) p(z)
$$

and

$$
1+\frac{1}{\tau}\left[\frac{(1-\mu)\left(w g^{\prime}(w)-g(w)\right)}{(1-\mu) g(w)+\mu w g^{\prime}(w)}\right]=\beta+(1-\beta) q(w)
$$


where $p(z)$ and $q(w)$ have the forms (2.8) and (2.9), respectively. By suitably comparing the coefficients in (2.17) and (2.18), we get

$$
\begin{gathered}
{\left[\frac{k(1-\mu)}{\tau}\right] a_{k+1}=(1-\beta) p_{k},} \\
\frac{2 k(1-\mu) a_{2 k+1}-k(1-\mu)(1+k \mu) a_{k+1}^{2}}{\tau}=(1-\beta) p_{2 k},
\end{gathered}
$$

and

$$
\begin{gathered}
-\left[\frac{k(1-\mu)}{\tau}\right] a_{k+1}=(1-\beta) q_{k} \\
\frac{2 k(1-\mu)\left[(k+1) a_{k+1}^{2}-a_{2 k+1}\right]-k(1-\mu)(1+k \mu) a_{k+1}^{2}}{\tau}=(1-\beta) q_{2 k} .
\end{gathered}
$$

From (2.19) and (2.21), we find

$$
p_{k}=-q_{k}
$$

and

$$
\frac{2 k^{2}(1-\mu)^{2} a_{k+1}^{2}}{\tau^{2}}=(1-\beta)^{2}\left(p_{k}^{2}+q_{k}^{2}\right) .
$$

Adding (2.20) and (2.22), we have

$$
\frac{2 k^{2}(1-\mu)^{2} a_{k+1}^{2}}{\tau}=(1-\beta)\left(p_{2 k}+q_{2 k}\right) .
$$

Applying Lemma 2.3, we obtain

$$
\left|a_{k+1}\right| \leq \frac{\sqrt{2|\tau|(1-\beta)}}{k(1-\mu)} .
$$

This is the bound on $\left|a_{k+1}\right|$ asserted in (2.16). In order to find the bound on $\left|a_{2 k+1}\right|$, by subtracting (2.22) from (2.20), we get

$$
\frac{2 k(1-\mu)\left[2 a_{2 k+1}-(k+1) a_{k+1}^{2}\right]}{\tau}=(1-\beta)\left(p_{2 k}-q_{2 k}\right),
$$

or equivalently,

$$
a_{2 k+1}=\frac{(k+1) a_{k+1}^{2}}{2}+\frac{\tau(1-\beta)\left(p_{2 k}-q_{2 k}\right)}{4 k(1-\mu)} .
$$

It follows from (2.23) and (2.24) that

$$
a_{2 k+1}=\frac{\tau^{2}(1-\beta)^{2}(k+1)\left(p_{k}^{2}+q_{k}^{2}\right)}{4 k^{2}(1-\mu)^{2}}+\frac{\tau(1-\beta)\left(p_{2 k}-q_{2 k}\right)}{4 k(1-\mu)} .
$$

Applying Lemma 2.3 once again for the coefficients $p_{k}, p_{2 k}, q_{k}$ and $q_{2 k}$, we easiliy obtain

$$
\left|a_{2 k+1}\right| \leq \frac{|\tau|^{2}(1-\beta)^{2}(k+1)}{2 k^{2}(1-\mu)^{2}}+\frac{|\tau|(1-\beta)}{k(1-\mu)} .
$$

For one-fold symmetric bi-univalent functions and $\tau=1$, Theorem 2.4 and Theorem 2.5 reduce to Corollary 2.8 and Corollary 2.9, respectively, which were proven very recently by Frasin [5] (see also [9]). 
Definition 2.6. A function $f \in \Sigma$ given by (1.1) is said to be in the class $\mathcal{N}_{\Sigma}(\mu, \alpha)$ if the following conditions are satisfied:

$$
\left|\arg \left(\frac{z f^{\prime}(z)}{(1-\mu) f(z)+\mu z f^{\prime}(z)}\right)\right|<\frac{\alpha \pi}{2}
$$

and

$$
\begin{gathered}
\left|\arg \left(\frac{w g^{\prime}(w)}{(1-\mu) g(w)+\mu w g^{\prime}(w)}\right)\right|<\frac{\alpha \pi}{2} \\
\quad(0<\alpha \leq 1 ; 0 \leq \mu<1 ; z, w \in \mathcal{U}),
\end{gathered}
$$

where the function $g=f^{-1}$ is given by (1.2).

Definition 2.7. A function $f \in \Sigma$ given by (1.1) is said to be in the class $\mathcal{N}_{\Sigma}(\mu, \beta)$ if the following conditions are satisfied:

$$
\operatorname{Re}\left(\frac{z f^{\prime}(z)}{(1-\mu) f(z)+\mu z f^{\prime}(z)}\right)>\beta
$$

and

$$
\begin{gathered}
\operatorname{Re}\left(\frac{w g^{\prime}(w)}{(1-\mu) g(w)+\mu w g^{\prime}(w)}\right)>\beta \\
(0 \leq \beta<1 ; 0 \leq \mu<1 ; z, w \in \mathcal{U}),
\end{gathered}
$$

where the function $g=f^{-1}$ is given by $(1.2)$.

Corollary 2.8. Let $f \in \mathcal{N}_{\Sigma}(\mu, \alpha)(0<\alpha \leq 1 ; 0 \leq \mu<1)$ be of the form (1.1). Then

$$
\left|a_{2}\right| \leq \frac{2 \alpha}{(1-\mu) \sqrt{1+\alpha}}
$$

and

$$
\left|a_{3}\right| \leq \frac{4 \alpha^{2}}{(1-\mu)^{2}}+\frac{\alpha}{1-\mu} .
$$

Corollary 2.9. Let $f \in \mathcal{N}_{\Sigma}(\mu, \beta)(0 \leq \beta<1 ; 0 \leq \mu<1)$ be of the form (1.1). Then

and

$$
\left|a_{2}\right| \leq \frac{\sqrt{2(1-\beta)}}{1-\mu}
$$

$$
\left|a_{3}\right| \leq \frac{(1-\beta)^{2}}{(1-\mu)^{2}}+\frac{1-\beta}{1-\mu} .
$$

If we set $\mu=0$ and $\tau=1$ in Theorem 2.4 and Theorem 2.5, then the classes $\mathcal{N}_{\Sigma_{k}}(\mu, \alpha)$ and $\mathcal{N}_{\Sigma_{k}}(\mu, \beta)$ reduce to the classes $\mathcal{N}_{\Sigma_{k}}^{\alpha}$ and $\mathcal{N}_{\Sigma_{k}}^{\beta}$ investigated recently by Srivastava et al. ([12]).

Definition 2.10. A function $f \in \Sigma_{k}$ given by (1.3) is said to be in the class $\mathcal{N}_{\Sigma_{k}}^{\alpha}(0<\alpha \leq 1)$ if the following conditions are satisfied:

$$
\left|\arg \left(\frac{z f^{\prime}(z)}{f(z)}\right)\right|<\frac{\alpha \pi}{2} \quad(z \in \mathcal{U})
$$

and

$$
\left|\arg \left(\frac{w g^{\prime}(w)}{g(w)}\right)\right|<\frac{\alpha \pi}{2} \quad(w \in \mathcal{U})
$$

and where the function $g$ is given by (1.4). 
Definition 2.11. A function $f \in \Sigma_{k}$ given by (1.3) is said to be in the class $\mathcal{N}_{\Sigma_{k}}^{\beta}(0 \leq \beta<1)$ if the following conditions are satisfied:

$$
\operatorname{Re}\left(\frac{z f^{\prime}(z)}{f(z)}\right)>\beta \quad(z \in \mathcal{U})
$$

and

$$
\operatorname{Re}\left(\frac{w g^{\prime}(w)}{g(w)}\right)>\beta \quad(w \in \mathcal{U}),
$$

and where the function $g$ is given by (1.4).

Corollary 2.12. Let $f \in \mathcal{N}_{\Sigma_{k}}^{\alpha}(0<\alpha \leq 1)$ be of the form (1.3). Then

$$
\left|a_{k+1}\right| \leq \frac{2 \alpha}{k \sqrt{1+\alpha}}
$$

and

$$
\left|a_{2 k+1}\right| \leq \frac{2 \alpha^{2}(k+1)}{k^{2}}+\frac{\alpha}{k}
$$

Corollary 2.13. Let $f \in \mathcal{N}_{\Sigma_{k}}^{\beta}(0 \leq \beta<1)$ be of the form (1.3). Then

$$
\left|a_{k+1}\right| \leq \frac{\sqrt{2(1-\beta)}}{k}
$$

and

$$
\left|a_{2 k+1}\right| \leq \frac{(1-\beta)^{2}(k+1)}{2 k^{2}}+\frac{1-\beta}{k}
$$

\section{REFERENCES}

[1] Ş. Altınkaya and S. Yalçın, Coefficient for certain subclasses of $m$-fold symmetric biunivalent function, Journal of Mathematics (2015), Article ID 241683, 5 pp.

[2] Ş. Altınkaya and S. Yalçın, On some subclasses of m-fold symmetric bi-univalent functions, Communications Faculty of Sciences University of Ankara Series A1 Mathematics and Statistics 67 (2018), 29-36.

[3] P. L. Duren, Univalent Functions, Grundlehren der Mathematischen Wissenschaften 259, Springer-Verlag, New York, Berlin, Heidelberg and Tokyo, 1983.

[4] S.S. Eker, Coefficient bounds for subclasses of m-fold symmetric bi-univalent functions, Turkish Journal of Mathematics 40 (2016), 641-646.

[5] B. A. Frasin, Coefficient bounds for certain classes of bi-univalent functions, Hacettepe Journal of Mathematics and Statistics 43 (2014), 383-389.

[6] Yu. E. Hohlov, Operators and operations in the class of univalent functions, Izvestiya Vysshikh Uchebnykh Zavedenii, Matematika 10 (1978), 83-89.

[7] W. Koepf, Coefficients of symmetric functions of bounded boundary rotations, Proceedings of the American Mathematical 105 (1989), 324-329.

[8] C. Pommerenke, On the coefficients of close-to-convex functions, Michigan Mathematical Journal 3 (1962), 259-269.

[9] H. M. Srivastava and D. Bansal, Coefficient estimates for a subclass of analytic and biunivalent functions, Journal of the Egyptian Mathematical Society 23 (2015), 242-246.

[10] H. M. Srivastava, S. Gaboury and F. Ghanim, Coefficient estimates for some subclasses of $m$-fold symmetric bi-univalent functions, Acta Universitatis Apulensis, Mathematics Informatics 41 (2015), 153-164.

[11] H. M. Srivastava, S. Gaboury and F. Ghanim, Initial coefficient estimates for some subclasses of m-fold symmetric bi-univalent functions, Acta Mathematica Scientia 36 (2016), 863-871. 
[12] H. M. Srivastava, S. Sivasubramanian and R. Sivakumar, Initial coefficient bounds for a subclass of m-fold symmetric bi-univalent functions, Tbilisi Mathematical Journal 7 (2014), 1-10.

[13] H. Tang, H. M. Srivastava, S. Sivasubramanian and P. Gurusamy, The Fekete-Szegö functional problems for some subclasses of $m$-fold symmetric bi-univalent functions, Journal of Mathematical Inequalities 10 (2016), 1063-1092.

Waggas Galib Atshan, Department of Mathematics, College of Science, University of ALQadisiyah, Diwaniyah-Iraq

e-mail: waggashnd@gmail.com, waggas.galib@qu.edu.iq

Sibel Yalçın, Department of Mathematics, Faculty of Arts and Sciences, Bursa Uludag University, 16059, Görükle, Bursa, Turkey

e-mail: syalcin@uludag.edu.tr

Rasha Abbas Hadi, Department of Mathematics, College of Science, University of AL-, Qadisiyah, Diwaniyah-Iraq

e-mail: rasha abbas.d@yahoo.com 